\title{
Association between healthy eating index- 2015 and various cognitive domains in US adults aged 60 years or older: the National Health and Nutrition Examination Survey (NHANES) 2011-2014
}

Yameng Fan', Yinyin Zhang ${ }^{1}$, Jiaqiao Li ${ }^{1}$, Yamei Liu' ${ }^{1}$, Huan Chang ${ }^{1}$, Yude Jiang ${ }^{1}$, Xingxia Tuo ${ }^{1}$, Long Zhou ${ }^{2^{*}}$ and Yan $\mathrm{Yu}^{1 *}$

\begin{abstract}
Background: Diet, as a modifiable factor, plays an important role in cognitive function. However, the association between adherence to the 2015-2020 Dietary Guidelines for Americans (DGA), measured by Healthy Eating Index (HEI)-2015, and cognitive function remains unclear. This study aims to explore whether HEl-2015 is associated with various cognitive domains and whether such association is modified by age, gender, or ethnicity in the US adults aged 60 years or older using data from the National Health and Nutrition Examination Survey (NHANES) 2011-2014.

Methods: HEl-2015 scores were calculated from 24-h dietary recall interviews. Cognitive function was evaluated by Digit Symbol Substitution Test (DSST, a measure of processing speed), Animal Fluency Test (AFT, a measure of executive function), a subtest from Consortium to Establish a Registry for Alzheimer's disease (CERAD, a measure of memory), and a composite-z score calculated by summing z scores of individual tests. The associations between HEl-2015 scores and cognitive performance were explored using multiple linear regression models.

Results: A total of 2450 participants aged 60 years or older were included. Participants with higher HEI-2015 scores were more likely to have higher DSST, AFT as well as composite-z scores $(P<0.05)$. Significant interaction effects were identified between HEl-2015 and ethnicity in specific cognitive domains $\left(P_{\text {interaction }}<0.05\right)$. Among HEl-2015 components, higher intakes of whole fruits and seafood and plant protein were associated with better cognitive performance $(P<0.05)$.
\end{abstract}

Conclusion: Higher adherence to DGA is associated with better cognitive performance, especially regarding processing speed and executive function among the US adults aged 60 years or older.

Keywords: Dietary guidance, HEl-2015, Cognitive function, Interaction

\footnotetext{
*Correspondence: wcuzhl@163.com; yanyupaper@yeah.net

2Department of Cardiology, Sichuan Provincial People's Hospital, University of Electronic Science and Technology of China, Chengdu, China

${ }^{1}$ School of Public Health, Xi'an Jiaotong University, 76 West Yanta Road, Xi'an,

Shaanxi 710061, People's Republic of China
}

(c) The Author(s). 2021 Open Access This article is licensed under a Creative Commons Attribution 4.0 International License, which permits use, sharing, adaptation, distribution and reproduction in any medium or format, as long as you give appropriate credit to the original author(s) and the source, provide a link to the Creative Commons licence, and indicate if changes were made. The images or other third party material in this article are included in the article's Creative Commons. licence, unless indicated otherwise in a credit line to the material. If material is not included in the article's Creative Commons licence and your intended use is not permitted by statutory regulation or exceeds the permitted use, you will need to obtain permission directly from the copyright holder. To view a copy of this licence, visit http://creativecommons.org/licenses/by/4.0/ The Creative Commons Public Domain Dedication waiver (http://creativecommons.org/publicdomain/zero/1.0/) applies to the data made available in this article, unless otherwise stated in a credit line to the data. 


\section{Background}

With the increase in lifespan, and the established association between cognitive decline and age itself across cognitive dimensions, it is becoming increasingly imperative to identify potential preventive behaviors for delaying cognitive decline. On this, diet, as a modifiable factor, is known to play an important role in cognitive function [1]. There is an urgent need for better understanding association between diet and cognition so that strategies can be improved to prevent and to better manage cognitive decline.

While there have been numerous studies examining the association of individual nutrients or foods with cognitive outcomes [2-4], recent approaches emphasize overall diet quality to account for nutrient interactions and reflect real-life dietary behaviors $[5,6]$. Diet quality is typically assessed using regional dietary patterns, such as with Mediterranean diet score, or national dietary guidelines, such as with Healthy Eating Index (HEI). As summarized in recent systemic reviews and metaanalyses, increasing evidence suggests that adherence to the Mediterranean diet shows a promising beneficial role on cognitive function [7, 8]. However, regional dietary patterns may not be appropriate in all national contexts. Studies on the associations between adherence to a national dietary guideline and cognitive outcomes are surprisingly sparse and the findings are varied. Some suggest that higher adherence to dietary guideline are associated with better cognitive performance, or reduced risk of cognitive impairment and dementia [9-12], while others are inconclusive [13-16].

Considering the differences in food culture and availability, we adopted the HEI-2015, which is used to assess adherence to the 2015-2020 Dietary Guidelines of Americans (DGA), to reflect the overall diet quality of the US population enrolled in the present study [17]. It was developed by the United States Department of Agriculture (USDA) and National Cancer Institute (NCI) and emphasizes high intake of total vegetables, greens and beans, total fruits, whole fruits, whole grains, dairy, total protein foods, seafood, plant proteins, and fatty acids while limiting the intake of sodium, refined grains, saturated fats, and added sugars. To date, there is no study evaluating the associations between HEI-2015 and multiple domains of cognitive performance in the US population.

Through this research, we want to answer whether aligning with 2015-2020 DGA, measured by HEI2015 , is related to various cognitive domains among US adults aged 60 years or older, and test whether the associations of HEI-2015 with cognitive domains vary by age, gender, or ethnicity using data from the National Health and Nutrition Examination Survey (NHANES) 2011-2014. We hypothesized that higher
HEI-2015 scores will be associated with better cognitive performance.

\section{Methods \\ Study population}

The data currently used are publicly available without personally identifiable information from NHANES (https://www.cdc.gov/nchs/nhanes/index.htm). A detailed description of the NHANES study design and methods is available elsewhere [18]. Briefly, NHANES is a program that administers ongoing 2-year-cycle crosssectional surveys, which is conducted by the Centers for Disease Control and Prevention (CDC). Based on a complex, multistage probability sampling design, NHANES examines a nationally representative sample of $\sim 5000$ persons each year selected from 15 different locations that are chosen from a sampling frame of all US counties. A major objective of NHANES is to estimate the health and nutritional status of the non-institutional population in the United States. In the present analysis, we combined 2 NHANES cycles (NHANES 2011-2012 and 2013-2014), because these two cycles specifically carried out a series of cognitive function testings. In total, 19,931 individuals participated in NHANES during 2011-2014, and our analysis was limited to 3632 adults aged 60 years or older who were eligible to complete the cognitive function testings. Among them, we excluded individuals with incomplete 24-h dietary recall data $(n=$ 564 ), extreme total energy intake of $<500$ or $>5000 \mathrm{kcal} /$ day for females, and $<500$ or $>8000 \mathrm{kcal} /$ day for males $(n=18)$, and incomplete cognitive function data $(n=$ 350). Moreover, individuals who had missing data on body mass index (BMI), sedentary time, blood pressure (BP), glycohemoglobin (HbA1c), and total cholesterol (TC) were further excluded $(n=250)$. Finally, 2450 individuals were included in our study (Fig. 1). Compared to the original population, study population was more likely to be younger (69.3 years VS 70.1 years), Non-Hispanic white, drinker, to have higher educational level and ratio of family income to poverty. No significant differences were observed in terms of other characteristics (Additional file 1).

\section{Development of HEl-2015 score}

Dietary intake data was collected from NHANES 24-h recall using the USDA automated multiple-pass method [19]. The 24-h dietary recall data were collected for 2 days, which were conducted by a trained interviewer face-to-face in the Mobile Examination Center on one day and a follow-up interview 3-10 days later via phone. Based on the two 24-h dietary recall data, food groups were determined using USDA Food Patterns Equivalence Database, and energy or nutrient content for food and 


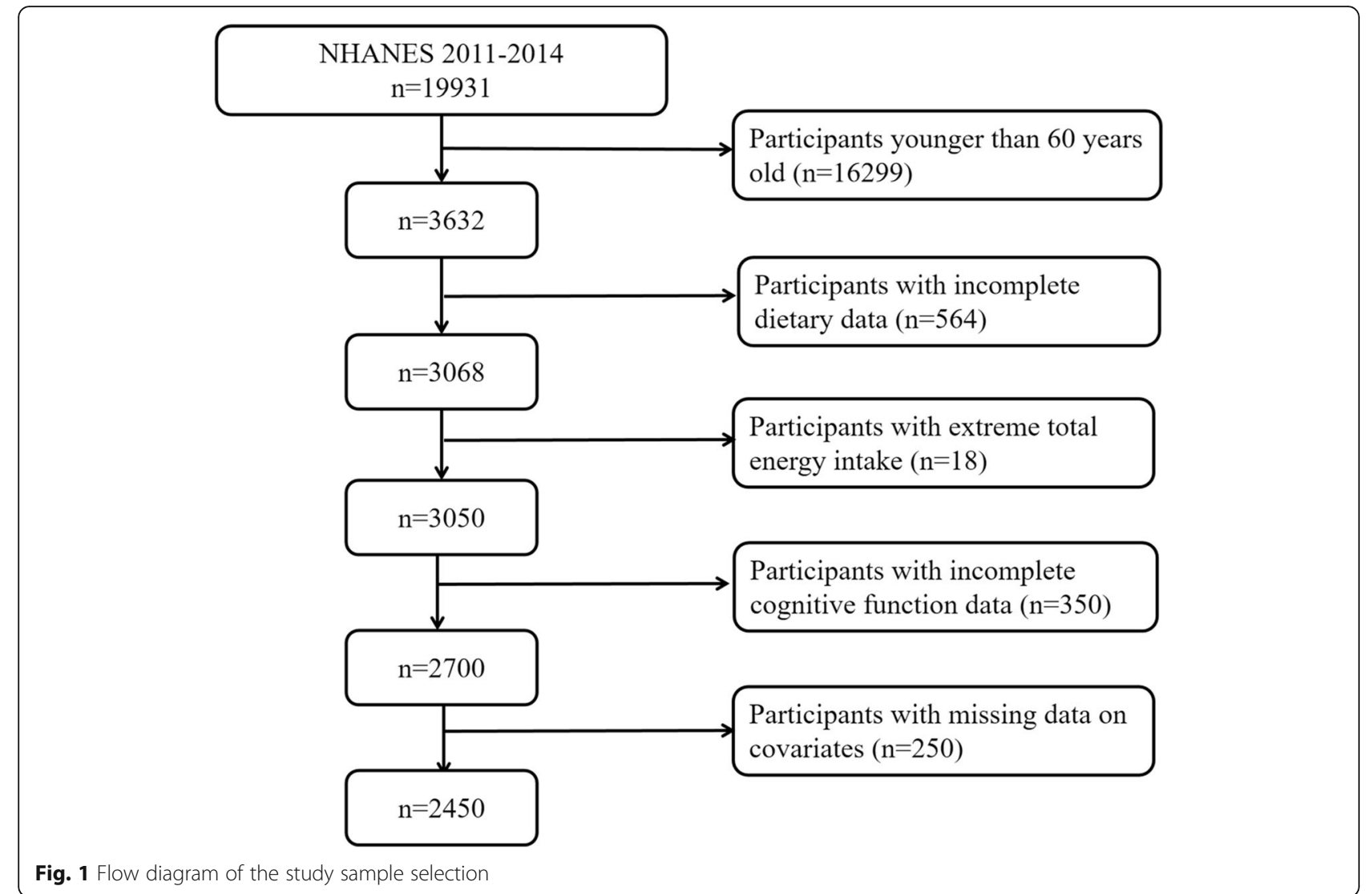

beverage recorded were computed using USDA Food and Nutrient Database for Dietary Studies.

HEI-2015 is a diet quality index developed in partnership by researchers from USDA and NCI to assess the adherence to 2015-2020 DGA and consists of 13 components (food groups or nutrients), including 9 adequacy components (total vegetables, greens and beans, total fruits, whole fruits, whole grains, dairy, total protein foods, seafood and plant proteins, and fatty acids) and 4 moderation components (sodium, refined grains, saturated fats, and added sugars) [20]. These components are scored based on an energy density of $1000 \mathrm{kcal}$, except for fatty acids which is a ratio of unsaturated to saturated fatty acids. The specific scoring standards for each component are shown in Additional file 2. Briefly, higher intakes result in higher scores for the adequacy components, while lower intakes result in higher scores for moderation components. The component scores are scored separately and summed to compute an overall score with a maximum of 100 (the higher the scores, the higher the compliance with 2015-2020 DGA).

\section{Assessment of cognitive function}

A series of cognitive function testings were performed among adults aged 60 years or older in NHANES 20112014, including Digit Symbol Substitution Test (DSST),
Animal Fluency Test (AFT), and the word learning subtest from the Consortium to Establish a Registry for Alzheimer's disease (CERAD). Participants who needed a proxy, did not pass the practice pretest, refused audio record, had communication problems, or gave up will have a missing score for the formal testings. In brief, DSST is used to assess the ability of processing speed [21]. The test provides a paper form that has a key at the top containing 9 numbers paired with symbols. Participants were asked to match corresponding symbols in the 133 boxes that adjoin the numbers in $120 \mathrm{~s}$. The score is the total number of correct matches. AFT is used to evaluate the categorical verbal fluency, a component of executive function, regardless of cultural context [22]. Participants were asked to name as many animals as possible in $60 \mathrm{~s}$. A point is given for each named ani$\mathrm{mal}$, and the final score represents the total number of correct named animals. CERAD word learning subtest was performed to assess both immediate and delayed memory, which consists of three consecutive learning trials and a delayed recall trial [23]. In the learning trials, participants were asked to read aloud 10 irrelevant words. The order of these 10 words is different in each of the three learning trials. After finishing the former two cognitive function testings (DSST and AFT), the delayed recall trial requiring participants to recall 10 words 
used in the learning trials which were conducted. In addition, a composite- $\mathrm{z}$ score was created by summing the $\mathrm{z}$ scores [(individual test score - mean score)/SD] of these three individual tests (DSST, AFT, CERAD). For all the tests, higher scores represent better cognitive performance.

\section{Covariates}

The NHANES release information about sociodemographic factors, health-related lifestyles, and health conditions through demographics, questionnaires, examination, and laboratory data. We included some of them as covariates that were thought to be related to cognitive function and/or diet quality based on the previous researches [24, 25]. Socio-demographic factors included age, gender (male and female), ethnicity (Hispanic, nonHispanic White, non-Hispanic Black, and Asian or other race), and education (less than high school, high school, and more than high school). Additionally, the ratio of family income to poverty was divided into three categories $(\leq 1.30,1.31-1.85,>1.85)$ to reflect house income. The higher ratio indicates better family financial situation. This index was calculated by dividing the family income by the Department of Health and Human Services' poverty guidelines, specific to family size, survey year and state [26].

As for health-related lifestyles, total time spent sitting except time sleeping (sedentary time) in a day was used as a potential physical activity indicator [27]. Smoker was defined as smoked at least 100 cigarettes in life [28]. Participants who drunk at least 12 times in the previous year were considered drinkers [29].

Concerning health conditions, the depressive symptom was assessed using 9-item Patient Health Questionnaire (PHQ-9). The score of each item ranges from 0 (not at all) to 3 (nearly every day), incorporating a total score with the maximum possible score of 27. Individuals with a PHQ-9 total score of 10 or greater were categorized with depressive symptoms [30]. BMI was calculated as weight $(\mathrm{kg})$ divided by height squared $(\mathrm{m})$ which were measured in Mobile Examination Center. Hypertension was defined as a SBP $\geq 140 \mathrm{mmHg}$ or a DBP $\geq 90 \mathrm{mmHg}$, and/or current use of antihypertensive medication [31]. Hypercholesterolaemia was defined as TC $\geq 240 \mathrm{mg} / \mathrm{dl}$ or current use of prescribed medicine for hypercholesterolaemia [32]. Participants were classified as having diabetes based on HbA1c $\geq 6.5 \%$ and/or current use with insulin or diabetic pills [33].

\section{Statistical analysis}

Characteristics of the study population are described as mean \pm standard deviation (SD) for continuous variables and number (percentage) for categorical variables. HEI2015 were categorized based on quartiles (quartile 1: < 25th percentile, quartile 2 : $\geq 25$ to 50 th percentile, quartile 3: $\geq 50$ to 75 th percentile, quartile $4: \geq 75$ th percentile). We tested differences in characteristics between groups with a one-way analysis of variance for continuous variables and with a chi-square test for categorical variables. Linear regression analyses were used to examine the associations between HEI-2015 scores, both as continuous variable and categorical variable (quartiles), and cognitive function. To test for trends across increasing quartiles of HEI-2015 scores, the median of HEI2015 scores in each quartile was calculated and used as a continuous variable. Model 1 was adjusted for age and gender; Model 2 was additionally adjusted for energy intake, ethnicity, BMI, drinking status, smoking status, sedentary time, education, ratio of family income to poverty, depressive symptom, hypertension, hypercholesterolaemia, and diabetes. To explore the dose-response relationship between HEI-2015 and cognitive function, we applied a restricted cubic spline with knots at the 5 th, 50th and 95th percentiles of the distribution of the exposure in a fully adjusted model (Model 2) using SAS macro "\%RCS_Reg" constructed by Desquilbet et al. [34]. We also performed a sensitivity analysis by restricting participants without a depressive symptom. Additionally, stratified analyses were conducted to test whether these associations differ by age groups (60-69 and 70 ), gender (male and female), or ethnicity (Hispanic, nonHispanic white, non-Hispanic black, Asian, or other race). For the exploratory purpose, we further examined the associations between HEI-2015 components (dichotomous) and domain-specific cognitive function adjusting for all covariates and other HEI-2015 components to identify whether statistically significant associations might be attributable to specific components. Data management and dose-response curve were performed using SAS version 9.4 (SAS Institute, Cary, North Carolina, USA) and other statistical analyses were performed using SPSS 24.0 (IBM, Armonk, NY, USA). A two-tailed $P$ value $<0.05$ was considered statistically significant.

\section{Results \\ Descriptive statistics}

The characteristics of the study population according to HEI-2015 quartiles are shown in Table 1. A total of 2450 adults aged 60 years or older were included for analysis. The mean age was $69.3 \pm 6.8$ years, and $49.8 \%$ of the participants were male. Participants with higher adherence to HEI-2015 were more likely to be older, female, Asian or other race, to have lower BMI, higher educational levels, and higher ratio of family income to poverty; while less likely to be smoker, non-Hispanic black race, diabetics, and to have depressive symptom $(P<0.05)$. No significant differences across HEI-2015 
Table 1 Characteristics of the overall target population according to HEl-2015 quartiles $(n=2450)^{1}$

\begin{tabular}{|c|c|c|c|c|c|}
\hline \multirow[b]{2}{*}{ Characteristics } & \multicolumn{5}{|c|}{ Quartiles of HEI-2015 } \\
\hline & $\overline{\mathrm{Q1}^{2}}$ & Q2 & Q3 & Q4 & \\
\hline N & 612 & 613 & 612 & 613 & $p^{3}$ \\
\hline Age (years) & $68.5 \pm 6.5$ & $69.2 \pm 6.8$ & $70.0 \pm 6.8$ & $69.8 \pm 6.8$ & $<0.001$ \\
\hline Gender, n (\%) & & & & & $<0.001$ \\
\hline Male & $348(56.9)$ & $327(53.3)$ & $286(46.7)$ & $258(42.1)$ & \\
\hline Female & $264(43.1)$ & $286(46.7)$ & $326(53.3)$ & $355(57.9)$ & \\
\hline Body mass index $\left(\mathrm{kg} / \mathrm{m}^{2}\right)$ & $29.8 \pm 6.9$ & $29.3 \pm 6.1$ & $28.9 \pm 5.9$ & $28.3 \pm 5.9$ & $<0.001$ \\
\hline Ethnicity, n (\%) & & & & & $<0.001$ \\
\hline Hispanic & $110(18.0)$ & $120(19.6)$ & $110(18.0)$ & $118(19.2)$ & \\
\hline Non-Hispanic white & $295(48.2)$ & $313(51.1)$ & $305(49.8)$ & $317(51.7)$ & \\
\hline Non-Hispanic black & $174(28.4)$ & $140(22.8)$ & $129(21.1)$ & $108(17.6)$ & \\
\hline Asian or other race & $33(5.4)$ & $40(6.5)$ & $68(11.1)$ & $70(11.4)$ & \\
\hline Education, n (\%) & & & & & $<0.001$ \\
\hline less than high school & $183(29.9)$ & $153(25.0)$ & $134(21.9)$ & $110(17.9)$ & \\
\hline high school & $170(27.8)$ & $159(25.9)$ & $141(23.0)$ & $107(17.5)$ & \\
\hline more than high school & $259(42.3)$ & $301(49.1)$ & $337(55.1)$ & $396(64.6)$ & \\
\hline Ratio of family income to poverty, $\mathrm{n}(\%)$ & & & & & $<0.001$ \\
\hline$\leq 1.30$ & $205(33.5)$ & $187(30.5)$ & $151(24.7)$ & $135(22.0)$ & \\
\hline $1.31 \sim 1.85$ & $126(20.6)$ & $104(17.0)$ & $105(17.2)$ & $106(17.3)$ & \\
\hline$>1.85$ & $281(45.9)$ & $322(52.5)$ & $356(58.2)$ & $372(60.7)$ & \\
\hline Energy intake (kcal/d) & $1876.8 \pm 748.4$ & $1850.2 \pm 691.4$ & $1800.7 \pm 661.3$ & $1782.0 \pm 618.4$ & 0.056 \\
\hline Sedentary time $(\mathrm{h} / \mathrm{d})$ & $6.6 \pm 3.3$ & $6.6 \pm 3.1$ & $6.5 \pm 3.2$ & $6.4 \pm 3.1$ & 0.603 \\
\hline Drinker, n (\%) & & & & & 0.241 \\
\hline Yes & $435(71.1)$ & $440(71.8)$ & $416(68.0)$ & $413(67.4)$ & \\
\hline No & $177(28.9)$ & $173(28.2)$ & $196(32.0)$ & $200(32.6)$ & \\
\hline Smoker, n (\%) & & & & & $<0.001$ \\
\hline Yes & $357(58.3)$ & $336(54.8)$ & $294(48.0)$ & $265(43.2)$ & \\
\hline No & $255(41.7)$ & $277(45.2)$ & $318(52.0)$ & $348(56.8)$ & \\
\hline Depressive symptom, n (\%) & & & & & 0.012 \\
\hline Yes & $61(10.0)$ & $63(10.3)$ & $55(9.0)$ & $34(5.5)$ & \\
\hline No & $551(90.0)$ & $550(89.7)$ & $557(91.0)$ & $579(94.5)$ & \\
\hline Hypertension, n (\%) & & & & & 0.339 \\
\hline Yes & $427(69.8)$ & $408(66.6)$ & $403(65.8)$ & $400(65.3)$ & \\
\hline No & $185(30.2)$ & $205(33.4)$ & $209(34.2)$ & $213(34.7)$ & \\
\hline Diabetes, n (\%) & & & & & 0.045 \\
\hline Yes & $180(29.4)$ & $168(27.4)$ & $151(24.7)$ & $140(22.8)$ & \\
\hline No & $432(70.6)$ & $445(72.6)$ & $461(75.3)$ & $473(77.2)$ & \\
\hline Hypercholesterolaemia, n (\%) & & & & & 0.903 \\
\hline Yes & $294(48.0)$ & $289(47.1)$ & $281(45.9)$ & $290(47.3)$ & \\
\hline No & $318(52.0)$ & $324(52.9)$ & $331(54.1)$ & $323(52.7)$ & \\
\hline
\end{tabular}

${ }^{1}$ Values are percentages (\%) or means \pm SD; HEl-2015: Healthy Eating Index-2015

${ }^{2} \mathrm{Q}$, quartiles; Q1 represents the unhealthiest diet quality, Q4 represents the healthiest diet quality

${ }^{3} P$-values refer to differences across tertiles and were calculated using chi-square tests for categorical variables and F-tests for continuous variables 
quartiles were observed in terms of sedentary time, energy intake, hypertension, and hypercholesterolaemia.

\section{Association between HEI-2015 score and cognitive function}

Table 2 presents the associations between HEI-2015 scores and cognitive performance. In an age and gender-adjusted Model 1, the highest HEI-2015 quartile was significantly associated with higher DSST score (B: $5.26 ; 95 \% \mathrm{CI}: 3.45,7.06 ; P<0.001)$, AFT score (B: $1.31 ; 95 \%$ CI: $0.71,1.91 ; P<0.001)$, CERAD score (B: 0.98 ; 95\%CI: $0.30,1.66 ; P<0.01$ ), and composite $\mathrm{z}$ score for cognitive function (B: 0.70 ; 95\%CI: 0.45 , 0.96; $P<0.001)$, compared to the lowest HEI-2015 quartile. There was evidence of a linear trend in all of these relations. When treating HEI-2015 as a continuous measure, each 1-unit increase in HEI-2015 score was associated with higher DSST score (B: 0.16; 95\%CI: 0.11, 0.21; $P<0.001)$, AFT score (B: 0.04; 95\%CI: $0.02,0.05 ; P<0.001)$, CERAD score (B: 0.03; 95\%CI: $0.01,0.05 ; P<0.01$ ), and composite $z$-score (B: 0.02; 95\%CI: $0.01,0.03 ; P<0.001)$. After fully adjusting in Model 2, the associations of HEI-2015 (highest versus lowest) with AFT (B: 0.65; 95\%CI: 0.09, 1.21; $P<$
0.05 ) and composite z-scores (B: 0.26; 95\%CI: 0.05, $0.47 ; P<0.05)$ attenuated but remained significant, whereas the associations with DSST and CERAD scores were no longer significant. We observed evidence of a linear trend in these associations of HEI2015 scores with DSST, AFT and composite z-scores except CERAD. In addition, each 1-unit increase in HEI-2015 was associated with DSST (B: 0.05; 95\% CI: 0.01, 0.09; $P<0.05$ ), AFT (B: 0.02; 95\% CI: 0.00, 0.03; $P<0.01$ ), and composite $\mathrm{z}$-scores (B: $0.01 ; 95 \% \mathrm{CI}$ : $0.00,0.01 ; P<0.01)$, but not associated with CERAD score.

Similar results were observed in sensitivity analyses (Additional file 3). When excluding individuals who suffered a depressive symptom $(n=177)$, HEI-2015 score (as a continuous variable) was still positively associated with DSST (B: 0.05; 95\% CI: 0.01, 0.09; $P<0.01$ ), AFT (B: 0.02; 95\% CI: 0.00, 0.03; $P<0.05)$ and composite $\mathrm{z}$-scores (B: $0.01 ; 95 \%$ CI: $0.00,0.01 ; P<0.05$ ) in fully adjusted model.

The dose-response relationship between HEI-2015 and domain-specific or composite $\mathrm{z}$-score cognitive function after fully adjusting are shown in Fig. 2. In restricted cubic spline models, HEI-2015 scores were positively

Table 2 Regression coefficients and 95\% confidence intervals of HEl-2015 for cognitive function scores $(n=2450)^{1}$

\begin{tabular}{|c|c|c|c|c|c|}
\hline & & \multicolumn{4}{|l|}{ Cognitive function } \\
\hline & & DSST & AFT & CERAD & Composite z-score \\
\hline HEl-2015 & $\mathrm{n}$ & B $(95 \% \mathrm{Cl})$ & B $(95 \% \mathrm{Cl})$ & B $(95 \% \mathrm{Cl})$ & B $(95 \% \mathrm{Cl})$ \\
\hline \multicolumn{6}{|l|}{ Model $1^{3}$} \\
\hline \multicolumn{6}{|l|}{ Categorical } \\
\hline $\mathrm{Q} 1^{4}$ & 612 & 0 (Reference) & 0 (Reference) & 0 (Reference) & 0 (Reference) \\
\hline Q2 & 613 & $0.58(-1.21,2.38)$ & $0.37(-0.23,0.97)$ & $0.08(-0.60,0.75)$ & $0.11(-0.14,0.37)$ \\
\hline Q3 & 612 & $2.45(0.65,4.25)^{* *}$ & $0.68(0.07,1.28)^{*}$ & $0.32(-0.35,1.01)$ & $0.32(0.07,0.57)^{*}$ \\
\hline Q4 & 613 & $5.26(3.45,7.06)^{* * * *}$ & $1.31(0.71,1.91)^{* * *}$ & $0.98(0.30,1.66)^{* *}$ & $0.70(0.45,0.96)^{* * *}$ \\
\hline$P_{\text {trend }}{ }^{5}$ & & $<0.001$ & $<0.001$ & 0.004 & $<0.001$ \\
\hline \multicolumn{6}{|l|}{ Continuous } \\
\hline 1 unit increase & & $0.16(0.11,0.21)^{* * *}$ & $0.04(0.02,0.05)^{* * *}$ & $0.03(0.01,0.05)^{* *}$ & $0.02(0.01,0.03)^{* * *}$ \\
\hline \multicolumn{6}{|l|}{ Model 2} \\
\hline Q1 & 612 & 0 (Reference) & 0 (Reference) & 0 (Reference) & 0 (Reference) \\
\hline Q2 & 613 & $-0.78(-2.16,0.60)$ & $0.09(-0.45,0.63)$ & $-0.14(-0.78,0.51)$ & $-0.05(-0.26,0.16)$ \\
\hline Q3 & 612 & $0.02(-1.38,1.42)$ & $0.34(-0.21,0.89)$ & $-0.06(-0.71,0.59)$ & $0.06(-0.16,0.27)$ \\
\hline Q4 & 613 & $1.40(-0.02,2.82)$ & $0.65(0.09,1.21)^{*}$ & $0.36(-0.30,1.02)$ & $0.26(0.05,0.47)^{*}$ \\
\hline$P_{\text {trend }}$ & & 0.031 & 0.015 & 0.273 & 0.011 \\
\hline \multicolumn{6}{|l|}{ Continuous } \\
\hline 1 unit increase & & $0.05(0.01,0.09)^{*}$ & $0.02(0.00,0.03)^{* *}$ & $0.01(-0.01,0.03)$ & $0.01(0.00,0.01)^{* *}$ \\
\hline
\end{tabular}

${ }^{1} \mathrm{HEl}$-2015: Healthy Eating Index; B: unstandardized regression coefficient; Cl: confidence interval; ${ }^{* * *} P<0.001,{ }^{* *} P<0.01,{ }^{*} P<0.05$

${ }^{2}$ The composite-z score was calculated by summing the $z$ scores ((test score - mean score)/SD) of the three individual tests

${ }^{3}$ Model 1: adjusted for age and gender; Model 2: Model 1 + daily energy intake, ethnicity, drinking status, smoking status, education, ratio of family income to poverty, sedentary time, depressive symptom, hypertension, hypercholesterolaemia, and diabetes

${ }^{4} \mathrm{Q}$, quartile; $\mathrm{Q} 1$ represents the unhealthiest diet quality, Q4 represents the healthiest diet quality

${ }^{5} P_{\text {trend: }}$ Test for trend based on a variable containing the median value for each quartile 


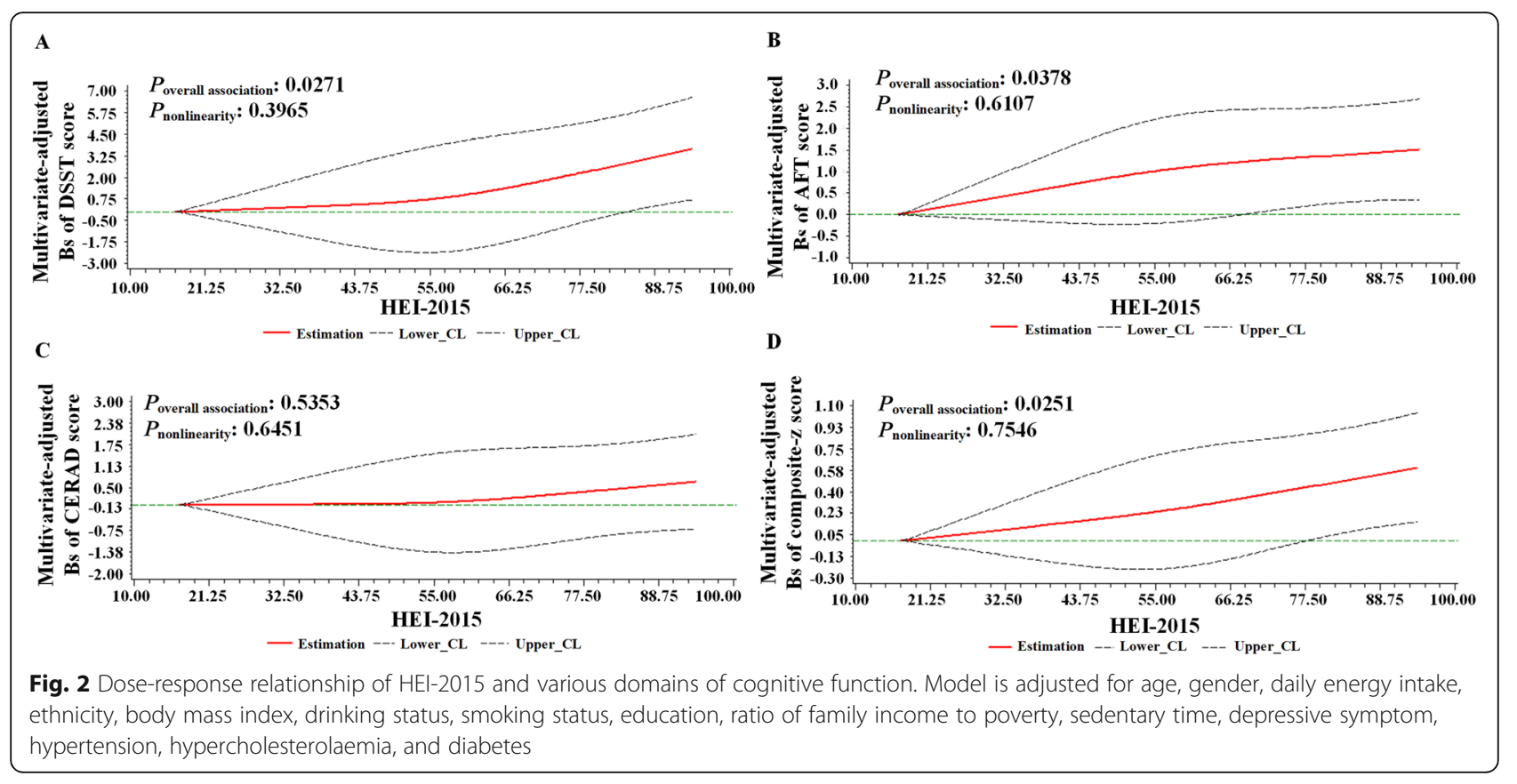

associated with DSST score $\left(P_{\text {nonlinearity }}=0.3965\right.$; Fig. $2 \mathrm{~A})$, AFT score $\left(P_{\text {nonlinearity }}=0.6107\right.$; Fig. $\left.2 \mathrm{~B}\right)$, and composite z-score $\left(P_{\text {nonlinearity }}=0.7546\right.$; Fig. $\left.2 \mathrm{C}\right)$ in a linear manner. However, no significant association was observed between HEI-2015 score and CERAD score in the fully adjusted model (Fig. 2D).

\section{Interaction effects}

Potential interaction effects were identified between HEI-2015 (treated as continuous) and ethnicity in relation to DSST score $\left(P_{\text {interaction }}<0.01\right.$; Fig. $\left.3 \mathrm{~A}\right)$ and AFT score $\left(P_{\text {interaction }}<0.05\right.$; Fig. $\left.3 \mathrm{~B}\right)$. HEI-2015 scores were positively associated with DSST (B: 0.08 ; 95\% CI: 0.03, $0.14 ; P<0.01$ ) and AFT scores (B: $0.04 ; 95 \%$ CI: 0.02 , $0.06 ; P<0.01)$, respectively, among the non-Hispanic white population in the fully adjusted model, but not among Hispanic, non-Hispanic blacks, and Asian or other race. In addition, no interaction effects were observed between age group or gender and HEI-2015 scores (treated as continuous) on any domain-specific cognitive function or composite- $z$ score in the fully adjusted model ( $P_{\text {interaction }}>0.05$; Fig. 3A-3D).

\section{Association between HEI-2015 components and cognitive function}

Given the significant associations between higher HEI2015 total scores and better performance on domainspecific cognitive function assessed by DSST and AFT, an exploratory analysis was performed to test the associations of each HEI-2015 component (dichotomous) with them, adjusting for all covariates included in Model 2 as well as the other components (Additional file 4). Among 13 HEI-2015 components, higher intakes of the whole fruits (B: 1.59; 95\% CI: 0.21, 2.98; $P<0.05$ ), seafood and plant protein (B: $1.46 ; 95 \% \mathrm{CI}: 0.34,2.58 ; P<0.05)$, and fatty acids (B: $1.28 ; 95 \% \mathrm{CI}: 0.08,2.48 ; P<0.05)$ were associated with better performance on DSST. Meanwhile, those with the higher consumption of whole fruits (B: 0.58; 95\% CI: 0.03, 1.12; $P<0.05)$ and seafood and plant protein (B: 0.78 ; 95\% CI: 0.34, 1.22; $P<0.001$ ) were more likely to have better performance on AFT.

\section{Discussion}

In this cross-sectional study, we found that adherence to 2015-2020 dietary guidelines for Americans, assessed by HEI-2015, is associated with higher DSST (a measure of processing speed), AFT (a measure of executive function), and composite-z scores in the US adults aged 60 years or older. Notably, the positive associations of HEI2015 with cognitive performance in the domains of processing speed and executive function are stronger among those of non-Hispanic white background. Additionally, exploratory analysis of components suggests adherence to the recommended intakes of whole fruits and seafood and plant protein were more likely to have better cognitive performance.

Our results are in line with studies that found associations between adherence to national dietary guidelines and cognitive outcomes. Higher adherence to the Dietary Guidelines for Americans, assessed by HEI-2005 or HEI-2010, has been reported to be associated with higher global cognition among Puerto Rican adults living 


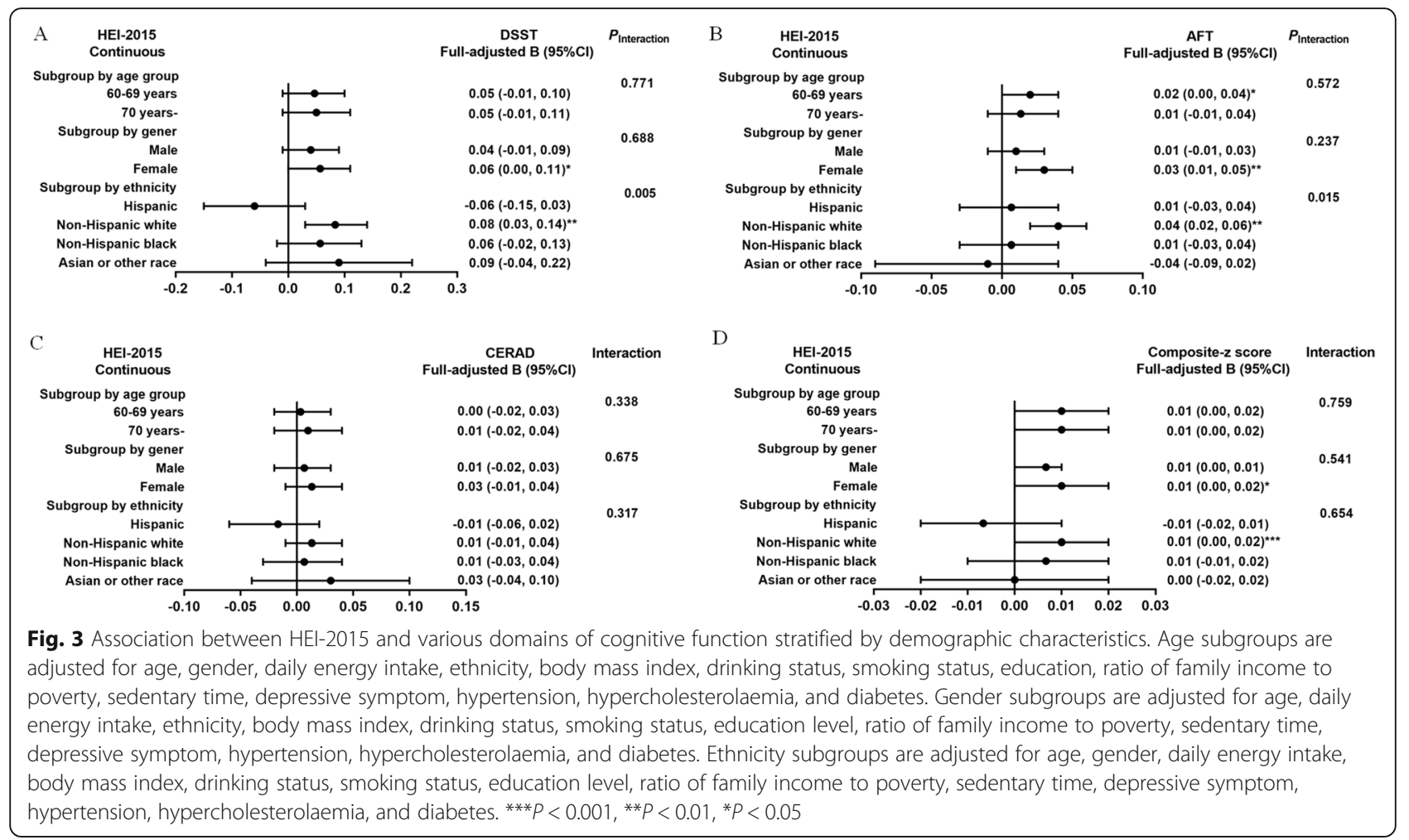

in Boston, and better cognitive performance for White and African Americans from Baltimore, respectively [10, 12]. Likewise, in a middle-aged and elderly Chinese population, the Chinese Dietary Guidelines Index-2018 was significantly associated with a lower risk of mild cognitive impairment [11]. However, our results are inconsistent with the Women's Health Initiative Memory Study that showed no significant associations between HEI-2010 and cognitive decline in older women [16]. Other studies conducted in Australia, Canada, and Chicago, US also reported that there was no association between adherence to a national dietary guideline and cognitive function [13-15]. These inconsistent results may emerge from variations in HEI components, study populations, or cognitive outcome measures, suggesting more research is needed to clarify the relationship between adherence to dietary patterns and cognitive outcomes. Prior to our study, we only found a relevant analysis from the Atherosclerosis Risk in Communities (ARIC) Study which reported that HEI-2015 was associated with a lower risk of incident dementia in black and white participants from four US communities [9]. Compared with the ARIC, our study enrolled multiethnic populations, assessed multiple cognitive domains, explored the interaction effects between HEI-2015 and some demographic factors, and included two important potential confounding factors: ratio of family income to poverty and depressive symptoms.
In the present study, HEI-2015 scores were not associated with memory sub-domain assessed by CERAD, but were positively associated with processing speed evaluated by DSST and executive function evaluated by AFT in the fully adjusted model, suggesting that some certain healthy dietary pattern may only have benefits for specific cognitive domains. Another analysis in the NHAN ES population revealed that the Mediterranean diet score was associated with memory and executive function rather than processing speed [35]. An analysis from the Hispanic Community Health Study found that adherence to an alternative to the HEI-2010 was associated with better verbal learning and memory, but not verbal fluency and processing speed [24]. The cognitive performance assessed by multiple cognitive function testings involving different domains may lead to inconsistent results on the relationship between diet and cognitive function. Thus, as many cognitive testings as possible should be used simultaneously to achieve a comprehensive analysis.

Notably, our findings are unique in showing that adherence to dietary guidance, as indicated by HEI-2015, was linked to cognitive performance in non-Hispanic White but not other races. Similar race/ethnicity differences have been observed in previous studies investigating associations between diet and cognition [36]. In the Einstein Aging Study, a healthy diet was related to executive dysfunction specifically in whites [37]. Similarly, 
the NHANES study showed that a protective role of adherence to the Mediterranean diet on cognition in nonHispanic White but not in other race/ethnic groups [35]. Current literature cannot clearly explain race/ethnicity differences in the diet-cognition associations. It is possible that statistical power was limited in the smaller sub-sample of minority groups. Among a large sample of middle-aged and older Hispanics/Latinos, an overall healthier diet quality has been found to be associated with better global cognition [24]. Additionally, differences in genetic or environmental risk factors and validity of dietary indices or cognitive testings between racial/ ethnic groups may also influence the association between diet and cognition.

Findings from our exploratory analyses suggest that adherence to the recommended intake of the whole fruits were more likely to have better performance on processing speed and executive function. This may be correlated to the protective effects of bioactive substances that are present in fruits, in particular flavonoids, vitamins, and carotenoids, which have anti-oxidant and anti-inflammatory properties [38]. Previous studies have shown that oxidative stress may contribute to the pathogenesis of Alzheimer's disease [39, 40]. Besides, the benefits of seafood and plant protein on cognition have also been observed in our study. Although it has been reported that higher intakes of seafood and plant protein were associated with better physical performance and lower mortality, there is limited evidence on the relation between seafood and plant protein and cognitive function, and the mechanism requires further study.

The present study has several advantages. First, NHANES enrolled multiethnic population with a relatively large sample size, making it possible to perform subgroup analysis according to age, gender, or ethnicity. To our knowledge, this is the first study to show the associations of HEI-2015 (the latest version of HEI) with various domains of cognitive function among a representative sample of the US population aged 60 years or older. Meanwhile, we conducted a dose-response analysis to assess the association between HEI-2015 score and cognitive performance. Additionally, a sensitivity analysis was performed by excluding participants who suffered depressive symptoms, and the results were stable. However, there are clear limitations that should also be considered. First, as a cross-sectional study design, we cannot ascertain a causal relationship. Second, NHANES did not collect data on cognitive impairment or neurocognitive disorders, thus, we were unable to take this into account when interpreting our findings. Third, the dietary data which was collected from the two days 24 recall, may not be able to reflect the usual intake well. Last but not least, some demographic characteristics of study samples were significantly different (albeit small) in the total samples. Thus, the results may not be generalized to the general population, and should be interpreted with caution.

\section{Conclusions}

We observed that adherence to the 2015-2020 dietary guideline for Americans, assessed by HEI-2015, is associated with cognitive performance, especially regarding processing speed and executive function in a US population aged 60 and over. The positive association between HEI-2015 score and cognitive performance is stronger in non-Hispanic White. Given the rational findings and several limitations in the present study, the results should be further validated in the large prospective cohort study.

\section{Abbreviations}

HEl: Healthy Eating Index; NHANES: The National Health and Nutrition Examination Survey; DSST: Digit Symbol Substitution Test; AFT: Animal Fluency Test; CERAD: Consortium to Establish a Registry for Alzheimer's disease; DGA: Dietary Guidelines for Americans; CDC: Centers for Disease Control and Prevention; USDA: United States Department of Agriculture; NCl: National Cancer Institute; PHQ: Patient Health Questionnaire; BP: Blood pressure; BMI: Body Mass Index; TC: Total cholesterol

\section{Supplementary Information}

The online version contains supplementary material available at https://doi. org/10.1186/s12889-021-11914-2.

Additional file 1. Comparison of characteristics between total and included participants.

Additional file 2. Components and scoring standards of HEl-2015².

Additional file 3. Sensitivity analysis on the associations between $\mathrm{HEI}$ 2015 (continuous) and cognition scores when excluding participants with depression symptom $(n=2273)^{1}$.

Additional file 4. Regression coefficients and 95\% confidence intervals of HEl-2015 components (dichotomous) for DSST and AFT $(n=2450)^{1}$.

\section{Acknowledgments}

We thank the public availability of NHANES data and thank all NHANES participants and staff for their valuable efforts.

\section{Authors' contributions}

YY, LZ and YMF contributed to the study design. LZ, YMF, YYZ and YDJ preformed the data analysis. YMF wrote the manuscript. JQL, YML, HC and XXT critically revised and edited the manuscript for important intellectual content. All authors reviewed and approved the final manuscript.

\section{Funding}

This work was not specifically funded.

\section{Availability of data and materials}

The datasets generated and/or analyzed during the current study are publicly available from the National Health and Nutrition Examination Survey website (https://www.cdc.gov/nchs/nhanes/index.htm).

\section{Declarations}

Ethics approval and consent to participate

All procedures performed in the study were in accordance with the Declaration of Helsinki. The study protocols for NHANES were approved by the National Center for Health Statistics (NCHS) Research Ethics Review Board (Protocol\#2011-17). All the participants signed the informed consent before participating in the study. 


\section{Consent for publication}

Not applicable.

\section{Competing interests}

The authors declare that they have no competing interests.

Received: 22 March 2021 Accepted: 27 September 2021

\section{Published online: 15 October 2021}

\section{References}

1. Smith PJ, Blumenthal JA. Dietary factors and cognitive decline. J Prev Alzheimers Dis. 2016;3(1):53-64. https://doi.org/10.14283/jpad.2015.71.

2. Cunnane SC, Plourde M, Pifferi F, Bégin M, Féart C, Barberger-Gateau P. Fish, docosahexaenoic acid and Alzheimer's disease. Prog Lipid Res. 2009;48(5): 239-56. https://doi.org/10.1016/j.plipres.2009.04.001.

3. Loef $M$, Walach $H$. Fruit, vegetables and prevention of cognitive decline or dementia: a systematic review of cohort studies. J Nutr Health Aging. 2012; 16(7):626-30. https://doi.org/10.1007/s12603-012-0097-x.

4. Wang Y, Lebwohl B, Mehta R, Cao Y, Green PHR, Grodstein F, et al. Longterm intake of gluten and cognitive function among US women. JAMA Netw Open. 2021;4(5):e2113020. https://doi.org/10.1001/jamanetworkopen.2 021.13020.

5. Alles B, et al. Dietary patterns: a novel approach to examine the link between nutrition and cognitive function in older individuals. Nutr Res Rev. 2012;25(2):207-22. https://doi.org/10.1017/S0954422412000133.

6. Scarmeas N, Anastasiou CA, Yannakoulia M. Nutrition and prevention of cognitive impairment. Lancet Neurol. 2018;17(11):1006-15. https://doi.org/1 0.1016/S1474-4422(18)30338-7.

7. Aridi YS, Walker JL, Wright O. The Association between the Mediterranean Dietary Pattern and Cognitive Health: A Systematic Review. Nutrients. 2017; 9(7):9(7). https://doi.org/10.3390/nu9070674

8. Loughrey DG, Lavecchia S, Brennan S, Lawlor BA, Kelly ME. The impact of the Mediterranean diet on the cognitive functioning of healthy older adults: a systematic review and Meta-analysis. Adv Nutr. 2017:8(4):571-86. https:// doi.org/10.3945/an.117.015495.

9. Hu EA, Wu A, Dearborn JL, Gottesman RF, Sharrett AR, Steffen LM, et al. Adherence to dietary patterns and risk of incident dementia: findings from the atherosclerosis risk in communities study. J Alzheimers Dis. 2020;78(2): 827-35. https://doi.org/10.3233/JAD-200392.

10. Wright RS, Waldstein SR, Kuczmarski MF, Pohlig RT, Gerassimakis CS, Gaynor $B$, et al. Diet quality and cognitive function in an urban sample: findings from the healthy aging in neighborhoods of diversity across the life span (HANDLS) study. Public Health Nutr. 2017;20(1):92-101. https://doi.org/10.1 017/S1368980016001361.

11. Zhang $X$, Wang Y, Liu W, Wang T, Wang L, Hao L, et al. Diet quality, gut microbiota, and microRNAs associated with mild cognitive impairment in middle-aged and elderly Chinese population. Am J Clin Nutr. 2021;114(2): 429-40. https://doi.org/10.1093/ajcn/nqab078.

12. Ye X, et al. Mediterranean diet, healthy eating index 2005, and cognitive function in middle-aged and older Puerto Rican adults. J Acad Nutr Diet. 2013;113(2):276-81.e13.

13. Tangney CC, Kwasny MJ, Li H, Wilson RS, Evans DA, Morris MC. Adherence to a Mediterranean-type dietary pattern and cognitive decline in a community population. Am J Clin Nutr. 2011;93(3):601-7. https://doi.org/1 0.3945/ajcn.110.007369.

14. Zabetian-Targhi F, Srikanth VK, Beare R, Moran C, Wang W, Breslin M, et al. Adherence to the Australian dietary guidelines is not associated with brain structure or cognitive function in older adults. J Nutr. 2020;150(6):1529-34. https://doi.org/10.1093/jn/nxaa052.

15. Shatenstein B, Ferland G, Belleville S, Gray-Donald K, Kergoat MJ, Morais J, et al. Diet quality and cognition among older adults from the NuAge study. Exp Gerontol. 2012;47(5):353-60. https://doi.org/10.1016/j.exger.2012.02.002.

16. Haring B, et al. No Association between Dietary Patterns and Risk for Cognitive Decline in Older Women with 9-Year Follow-Up: Data from the Women's Health Initiative Memory Study. J Acad Nutr Diet. 2016;116(6):921930.e1.

17. Krebs-Smith SM, Pannucci TRE, Subar AF, Kirkpatrick SI, Lerman JL, Tooze JA, et al. Update of the healthy eating index: HEl-2015. J Acad Nutr Diet. 2018; 118(9):1591-602. https://doi.org/10.1016/j.jand.2018.05.021.

18. Zipf G, et al. National health and nutrition examination survey: Plan and operations, 1999-2010. Vital Health Stat 1. 2013(56):1-37.
19. Moshfegh AJ, Rhodes DG, Baer DJ, Murayi T, Clemens JC, Rumpler WV, et al. The US Department of Agriculture Automated Multiple-Pass Method reduces bias in the collection of energy intakes. Am J Clin Nutr. 2008;88(2): 324-32. https://doi.org/10.1093/ajcn/88.2.324.

20. Reedy J, Lerman JL, Krebs-Smith SM, Kirkpatrick SI, Pannucci TRE, Wilson MM, et al. Evaluation of the healthy eating Index-2015. J Acad Nutr Diet. 2018;118(9):1622-33. https://doi.org/10.1016/j.jand.2018.05.019.

21. Linghui D, Shi Q, Chi C, Xiaolei L, Lixing Z, Zhiliang Z, et al. The association between leukocyte telomere length and cognitive performance among the American elderly. Front Aging Neurosci. 2020;12:527658. https://doi.org/1 0.3389/fnagi.2020.527658.

22. Cardoso BR, Hare DJ, Macpherson H. Sex-dependent association between selenium status and cognitive performance in older adults. Eur J Nutr. 2021; 60(2):153-59. https://doi.org/10.1007/s00394-020-02384-0.

23. Frith E, Shivappa N, Mann JR, Hébert JR, Wirth MD, Loprinzi PD. Dietary inflammatory index and memory function: population-based national sample of elderly Americans. Br J Nutr. 2018;119(5):552-8. https://doi.org/1 $0.1017 /$ S0007114517003804.

24. Estrella ML, Durazo-Arvizu RA, Mattei J, Mossavar-Rahmani Y, Perreira KM, Siega-Riz AM, et al. Alternate healthy eating index is positively associated with cognitive function among middle-aged and older Hispanics/Latinos in the HCHS/SOL. J Nutr. 2020;150(6):1478-87. https://doi.org/10.1093/jn/nxaa 023.

25. Tsai CK, Kao TW, Lee JT, Wu CJ, Hueng DY, Liang CS, et al. Increased risk of cognitive impairment in patients with components of metabolic syndrome. Medicine (Baltimore). 2016;95(36):e4791. https://doi.org/10.1097/MD. 0000000000004791.

26. Taylor MK, Mahnken JD, Sullivan DK, NHANES. Reveals cognition of US older adults may benefit from better adaptation to the Mediterranean diet. Nutrients. 2011-2014;2020(7):12(7). https://doi.org/10.3390/nu12071929.

27. Edwards MK, Loprinzi PD. Combined associations of sedentary behavior and cardiorespiratory fitness on cognitive function among older adults. Int J Cardiol. 2017;229:71-4. https://doi.org/10.1016/j.ijcard.2016.11.264.

28. Pereira $\mathrm{L}$, et al. Relationship between urinary nitrate concentrations and cognitive function in older adults: findings from the NHANES survey. Int J Food Sci Nutr. 2021:1-11.

29. Dong X, Li S, Sun J, Li Y, Zhang D. Association of Coffee, Decaffeinated Coffee and Caffeine Intake from Coffee with Cognitive Performance in Older Adults: National Health and Nutrition Examination Survey (NHANES) 2011-2014. Nutrients. 2020;12(3):12(3). https://doi.org/10.3390/nu12030840.

30. Lin S, Shen Y. Dietary carotenoids intake and depressive symptoms in US adults, NHANES 2015-2016. J Affect Disord. 2021;282:41-5. https://doi.org/1 0.1016/j.jad.2020.12.098.

31. Zhou L, Yang Y, Feng $Y$, Zhao X, Fan $Y$, Rong J, et al. Association between dietary sodium intake and non-alcoholic fatty liver disease in the US population. Public Health Nutr. 2021;24(5):993-1000. https://doi.org/10.1017/ S136898001900483X.

32. Lee SH, Gong HS, Kim S, Kim J, Baek GH. Is there a relation between lateral epicondylitis and Total cholesterol levels? Arthroscopy. 2019;35(5):1379-84. https://doi.org/10.1016/j.arthro.2019.01.048.

33. Zhou L, Li X, Li S, Wen X, Peng Y, Zhao L. Relationship between dietary choline intake and diabetes mellitus in the National Health and nutrition examination survey 2007-2010. J Diabetes. 2021;13(7):554-61. https://doi. org/10.1111/1753-0407.13143.

34. Desquilbet L, Mariotti F. Dose-response analyses using restricted cubic spline functions in public health research. Stat Med. 2010;29(9):1037-57. https://doi.org/10.1002/sim.3841.

35. Gu Y, Guo J, Moshfegh AJ. Race/ethnicity and gender modify the association between diet and cognition in U.S. older adults: National Health and Nutrition Examination Survey 2011-2014. Alzheimers Dement (N Y). 2021;7(1):e12128.

36. Agarwal P, Morris MC, Barnes LL. Racial differences in dietary relations to cognitive decline and Alzheimer's disease risk: do we know enough? Front Hum Neurosci. 2020;14:359. https://doi.org/10.3389/fnhum.2020. 00359.

37. Sundermann EE, Katz MJ, Lipton RB, Lichtenstein AH, Derby CA. A brief dietary assessment predicts executive dysfunction in an elderly cohort: results from the Einstein aging study. J Am Geriatr Soc. 2016;64(11):e131-6. https://doi.org/10.1111/jgs.14381.

38. Wu L, Sun D, Tan Y. Intake of fruit and vegetables and the incident risk of cognitive disorders: a systematic review and Meta-analysis of cohort studies. 
J Nutr Health Aging. 2017;21(10):1284-90. https://doi.org/10.1007/s12603-01 7-0875-6.

39. Christen Y. Oxidative stress and Alzheimer disease. Am J Clin Nutr. 2000; 71(2):621S-9S. https://doi.org/10.1093/ajcn/71.2.621s.

40. Hajjar I, Hayek SS, Goldstein FC, Martin G, Jones DP, Quyyumi A. Oxidative stress predicts cognitive decline with aging in healthy adults: an observational study. J Neuroinflammation. 2018;15(1):17. https://doi.org/1 0.1186/s12974-017-1026-z.

\section{Publisher's Note}

Springer Nature remains neutral with regard to jurisdictional claims in published maps and institutional affiliations.

Ready to submit your research? Choose BMC and benefit from:

- fast, convenient online submission

- thorough peer review by experienced researchers in your field

- rapid publication on acceptance

- support for research data, including large and complex data types

- gold Open Access which fosters wider collaboration and increased citations

- maximum visibility for your research: over $100 \mathrm{M}$ website views per year

At $\mathrm{BMC}$, research is always in progress. 